International Journal of Foundations of Computer Science

(C) World Scientific Publishing Company

\title{
MERGEABLE DOUBLE-ENDED PRIORITY QUEUES *
}

\author{
SEONGHUN CHO \\ Synopsis Inc \\ 700 Middlefield Road \\ Mountain View, CA 94043 \\ seonghun@synopsis.com \\ and \\ SARTAJ SAHNI \\ CISE Department, University of Florida, \\ Gainesville, FL 32611, USA \\ sahni@cise.ufl.edu \\ Received 2 April 1998 \\ Revised 29 July 1998 \\ Communicated by O. H. Ibarra
}

\begin{abstract}
We show that the leftist tree data structure may be adapted to obtain data structures that permit the double-ended priority queue operations Insert, DeleteMin, DeleteMax, and Merge to be done in $O(\log n)$ time where $n$ is the size of the resulting queue. The operations FindMin and FindMax can be done in $O(1)$ time. Experimental results are also presented.

Keywords: Double-ended priority queues, complexity, performance.
\end{abstract}

\section{Introduction}

A double-ended priority queue (DEPQ) is a collection of elements each of which has a priority. The operations that may be performed on a DEPQ are FindMax (find the element with maximum priority), FindMin (find the element with minimum priority), Insert (insert a new element into the DEPQ), DeleteMax (delete an element with maximum priority), and DeleteMin (delete an element with minimum priority).

Several simple and efficient implicit data structures have been proposed for the representation of a DEPQ [13, 2, 4, 10, 5, 9]. In all of these, FindMax and FindMin take $O(1)$ time and the remaining operations take $O(\log n)$ time each $(n$ is the number of elements in the DEPQ). 0111 . 
The twin heaps of Ref. 13, the min-max pair heaps of Ref. 10, the interval heaps of Ref. 9, and the diamond deques of Ref. 5 are virtually identical data structures. In each of these structures, an $n$ element DEPQ is represented by a min heap with $\lceil n / 2\rceil$ elements and a max heap with the remaining $\lfloor n / 2\rfloor$ elements. The two heaps satisfy the property that each element in the min heap is $\leq$ the corresponding element in the max heap. When the number of elements in the DEPQ is odd, the min heap has one element (i.e., element $\lceil n / 2\rceil$ ) that has no corresponding element (recall that a heap is a complete binary tree [8] and two elements correspond if they occupy the same position in their respective binary trees) in the max heap. In the twin heaps of Ref. 13, this is handled as a special case and one element is kept outside of the two heaps. In min-max pair heaps, interval heaps, and diamond deques, the case when $n$ is odd is handled by requiring element $\lceil n / 2\rceil$ of the min heap to be $\leq$ element $\lfloor n / 4\rfloor$ of the max heap.

In the twin heaps of Ref. 13, the min and max heaps are stored in two arrays $M I N$ and $M A X$ using the standard array representation of a complete binary tree [8]. The correspondence property becomes $M I N[i] \leq M A X[i], 1 \leq i \leq\lfloor n / 2\rfloor$. In the min-max pair heaps of Ref. 10 and the interval heaps of Ref. 9, the two heaps are stored in a single array $M I N M A X$ and we have MINMAX [i].min being the $i$ 'th element of the min heap, $1 \leq i \leq\lceil n / 2\rceil$ and $M I N M A X[i]$.max being the $i$ 'th element of the max heap, $1 \leq i \leq\lfloor n / 2\rfloor$. In the diamond deque [5], the two heaps are mapped into a single array with the min heap occupying even positions (beginning with position 0) and the max heap occupying odd positions (beginning with position 1). Since this mapping is slightly more complex than the ones used in twin heaps, min-max pair heaps, and interval heaps, actual implementations of the diamond deque are expected to be slightly slower than implementations of the remaining three structures.

In the min-max heap structure [2], all $n$ DEPQ elements are stored in a complete binary tree with alternating levels being min levels and max levels [8]. This structure is more complex than the ones mentioned above and so is expected to run slower than those by a constant factor. The deap structure of Ref. 4 is similar to the two heap structures of Refs. 13,10,9,5. At the conceptual level, we have a min heap and a max heap. However, the distribution of elements between the two is not $\lceil n / 2\rceil$ and $\lfloor n / 2\rfloor$. Rather, we begin with an $n+1$ node complete binary tree. Its left subtree is the min heap and its right the max heap. The correspondence property is more involved as several leaf elements of the min heap may not have a corresponding element in the max heap. Let $i$ be a leaf of the min heap. Let $j$ be the corresponding position in the max heap. Element $i$ of the min heap is required to be $\leq$ element $j$ of the max heap. If the max heap has no element at position $j$, then element $i$ of the min heap is to be $\leq$ element $\lfloor j / 2\rfloor$ of the max heap. While deaps are faster than min-max heaps (by a constant factor), they are slower than twin heaps, min-max pair heaps, and interval heaps.

A mergeable DEPQ (MDEPQ) supports all of the operations supported by a DEPQ as well as the operation Merge which merges (combines) two DEPQs into one. To merge two DEPQs in less than linear time, it is essential that the DEPQs 
be represented using explicit pointers (rather than implicit ones as in the array representation of a heap) as otherwise a linear number of elements need to be moved from their initial to their final locations. Olariu et al. [10] have shown that when the min-max pair heap is represented in such a way, an $n$ element DEPQ may be merged with a $k$ element one $(k \leq n)$ in $O(\log (n / k) * \log k)$ time. When $k$ $=\sqrt{n}$, this is $O\left(\log ^{2} n\right)$. For the remaining DEPQ structures, no sublinear Merge method is known. In fact, Hasham and Sack [7] have shown that the complexity of merging two min-max heaps of size $n$ and $k$, respectively, is $\Omega(n+k)$. Brodal [3] has developed an MDEPQ implementation that allows one to find the min and max elements, insert an element, and merge two priority queues in $\mathrm{O}(1)$ time. The time needed to delete the minimum or maximum element is $\mathrm{O}(\log n)$. Although the asymptotic complexity provided by this data structure are the best one can hope for [3], the data structure has practical limitations. First, each element is represented twice using a total of 16 fields per element. Second, even though the delete operations have $\mathrm{O}(\log n)$ complexity, the constant factors are very high and the data structure will not perform well unless find, insert, and merge are the primary operations.

In this paper, we show that leftist trees $[6,8,12]$ may be adapted to obtain a simple representation for MDEPQs in which Merge takes $O(\log n k)=O(\log n)$ time and the remaining operations have the same asymptotic complexity as when any of the aforementioned DEPQ representations is used. In Section 2, we present a simple adaption that does this. This is refined, in Section 3, to obtain a more space efficient adaptation. The adaptation of Section 3, the twin leftist tree, represents each element just once and requires 6 fields per element (in contrast to the 16 fields required by Brodal's structure). Experimental results are presented in Section 4 . These results show that our data structure is very practical. In fact, it outperforms the data structures deveoped earlier for DEPQs even on operation mixes that do not contain any merge operations.

\section{First Adaptation Of Leftist Trees}

Crane [6] has proposed a simple data structure, the leftist tree, for the representation of single ended mergeable priority queues. Let $T$ be a binary tree. The extended binary tree $T^{\prime}$ corresonding to $T$ is obtained by replacing each null child of $T$ by an external node. For any node $x$ in $T$, let $\operatorname{sh}(x)$ be the length of a shortest path from $x$ to an external node in the subtree of $T^{\prime}$ rooted at $x$. Let $\operatorname{sh}(x)=0$ for $x$ an external node. $T$ is a leftist tree iff $\operatorname{sh}($ leftchild $x) \geq \operatorname{sh}($ rightchild $x)$ for all nodes $x$ of $T$. Let $\operatorname{sh}(T)=\operatorname{sh}($ root of $T)$.

Lemma 1 (Refs. 6,8,12) For any $n$ node leftist tree $T, s h(T)=O(\log n)$ and the rightmost root to external node path has length $\operatorname{sh}(T)$.

In a min $(\max )$ leftist tree, the priority of the element in each node $x$ is $\leq(\geq)$ that of all elements in the subtree rooted at $x$. In a min (max) leftist tree, FindMin (FindMax) can be done in $O(1)$ time, Insert and DeleteMin (DeleteMax) take $O(\log n)$ time, and a merge takes $O(\log n k)$ time.

A simple way to use leftist trees to represent an MDEPQ is to simultaneously 
maintain both a min and a max leftist tree of all $n$ MDEPQ elements. Figure 1 shows an example representation for the case when $n=7$ and the element priorities are $(1,2,4,5,5,6,8)$. Let us call this representation of an MDEPQ the dual leftist tree representation.

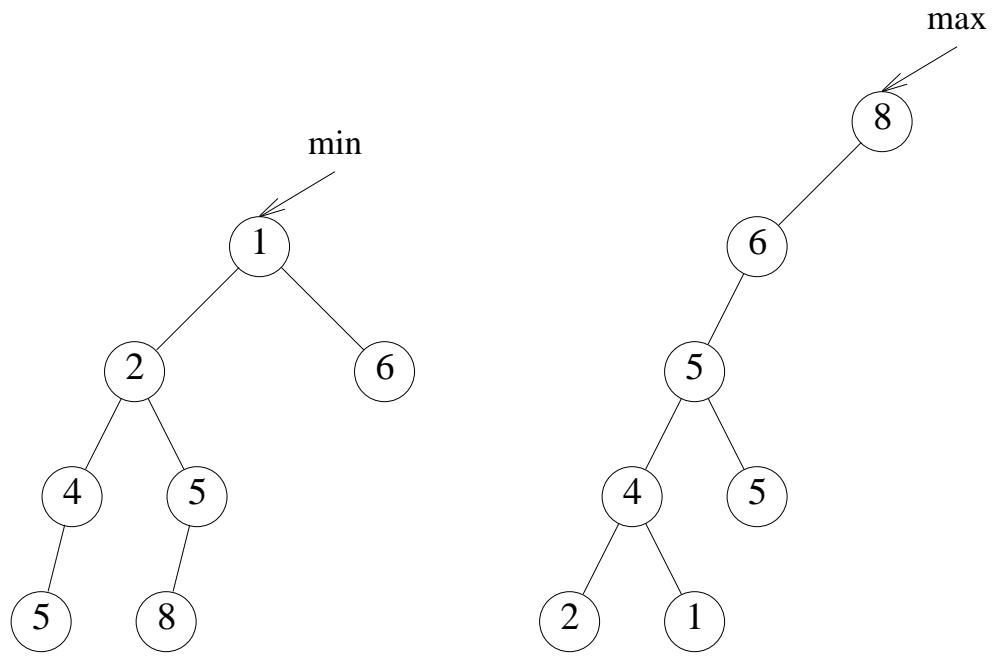

Fig. 1. Dual leftist trees

We obtain a space efficient representation of a dual leftist tree when we use nodes with the fields: $L 1$ (left child for min leftist tree), $L 2$, (left child for max leftist tree) $R 1$ (right child for min leftist tree), $R 2$ (right child for max leftist tree), $P 1$ (parent for min leftist tree), $P 2$ (parent for max leftist tree), $\operatorname{sh} 1$ (sh() value for min leftist tree), $\operatorname{sh} 2(\operatorname{sh}()$ value for max leftist tree), and data. $L 1, R 1, P 1$, and $\operatorname{sh} 1(L 2, R 2, P 2$, and $s h 2)$ are used to form the min $(\max )$ leftist tree. $L 1(R 1, P 1)$ points to the left child (right child, parent) of the node in the min leftist tree. $\operatorname{sh} 1$ $(\operatorname{sh} 2)$ is the $s h$ value of the node when viewed as a member of the min $(\max )$ leftist tree. With this node structure, the dual leftist tree representation of an $n$ element MDEPQ requires space for $n$ data fields, $6 n$ pointer fields, and $2 n s h$ fields.

Let $Q$ be an MDEPQ represented as a dual leftist tree. Let Q.min and Q.max, respectively, point to the min and max leftist trees of $Q$. The MDEPQ FindMax (FindMin) operation is done in $O(1)$ time by simply invoking the max (min) leftist tree FindMax (FindMin) operation on the leftist tree Q.max (Q.min). To insert a new item into $Q$, we insert it into Q.max and Q.min using the $O(\log n)$ time codes to insert into a max and min leftist tree. Hence, the $M D E P Q$ insert operation takes $O(\log n)$ time. To merge an MDEPQ $Q 1$ with $n$ elements with an MDEPQ $Q 2$ with $k$ elements, we merge Q1.min with $Q 2 . \min$ and $Q 1$.max with Q2.max. Each of these merges takes $O(\log n k)$ time. So, the two $M D E P Q$ s may be merged in $O(\log n k)$ time. For the MDEPQ DeleteMin (DeleteMax) operation, let $x$ be the node at the root of the $\min (\max )$ leftist tree Q.min $(Q . \max )$. We need to perform 
a DeleteMin (DeleteMax) in the $\min (\max )$ leftist tree and also delete node $x$ from the max ( $\min$ ) leftist tree. Since DeleteMax (DeleteMin) is a standard max (min) leftist tree operation that takes $O(\log n)$ time, we need only be concerned with the deletion of an arbitrary node $x$ from a max and a min tree. Figure 2 shows how to do this from the min leftist tree Q.min. The notation $x \rightarrow P 1$ means the $P 1$ filed of the node pointed at by $x$. The algorithm for deletion of $x$ from a max leftist tree is similar.

Step 1: if $(x==$ Q.min $)$ do a min leftist tree DeleteMin on Q.min; return;

Step 2: $p=x \rightarrow P 1 ; / /$ parent of $x$ in min leftist tree

Step 3: Change appropriate child field ( $L 1$ or $R 1$ ) of $p$ from $x$ to $x \rightarrow L 1$.

Follow path from $p$ towards root of min leftist tree using $P 1$ pointers and swapping subtrees ( $L 1$ and $R 1)$ as needed to ensure leftist tree property and adjusting $s h 1$ values.

Stop when this has been done at the root or when we reach a node whose $\operatorname{sh} 1$ value doesn't change.

Step 4: Merge the min leftist trees Q.min and $x \rightarrow R 1$ ( $x \rightarrow R 1$ may be null).

Step 5: Delete the node $x$;

Fig. 2. Deletion of node $x$ from the min leftist tree Q.min

For the complexity of this algorithm, we note that steps 1,2, 4, and 5 together take $O(\log n)$ time. For Step 3, note that even though the path from $p$ to the root may be $O(n)$ long, only $O(\log n)$ nodes on this path can be reached as the reached nodes (except possibly the last one) have increasing $s h 1$ value following Step 3 and the maximum $\operatorname{sh} 1$ value is $O(\log n)$.

\section{Second Adaptation Of Leftist Trees}

\subsection{Strategy}

This adaptation is called the twin leftist tree adaptation. We borrow ideas used in the twin heaps representation of Ref. 13. At most one of the $n$ MDEPQ elements will be kept in a buffer and the remaining elements distributed between a min $(M i n L T)$ and a $\max (M a x L T)$ leftist tree. Elements in the min and max leftist trees satisfy the following correspondence property:

For each element $e$ in $\operatorname{Min} L T$ ( $\operatorname{Max} L T)$, there is at least one element in $\operatorname{Max} L T(\operatorname{Min} L T)$ whose priority is $\geq(\leq)$ that of $e$.

As a result of this correspondence property, the element with least (max) priority is either the one (if any) in the buffer or the one in the root of MinLT (MaxLT). By comparing these two elements, FindMin (FindMax) can be accomplished in $O(1)$ time. 
Each leftist tree node has the following fields: $L, R, P, C$, sh, and data. $L$ $(R, P)$ points to the left child (right child, parent); $C$ points to a node (called the corresponding node) in the other leftist tree; and $s h$ is as defined for a leftist tree. For any node $x$ in the min $(\max )$ leftist tree, $x \rightarrow C$ is either $N U L L$ or points to a node in the $\max (\min )$ leftist tree that has priority $\geq(\leq)$ that of $x$. Furthermore, if $x \rightarrow C$ is not null, then $x$ equals $x \rightarrow C \rightarrow C$.

There are several ways $[13,4,8]$ in which we can enforce the correspondence property. Two of these are given below:

1. Total Correspondence. In this, no node in either MaxLT or MinLT has a null correspondence pointer $C$. As a result of this and the requirement $x \rightarrow C \rightarrow C$ equal $x$ for every node with a non-null $C$ pointer, the number of nodes in MaxLT equals that in MinLT. An example total correspondence twin leftist tree with $n=7$ and priorities $(1,2,4,5,5,6,8)$ is shown in Figure 3(a).

2. Leaf Correspondence. In this, no leaf of MinLT or MaxLT may have a null pointer. Figure 3(b) gives an example of a leaf correspondence twin leftist tree.

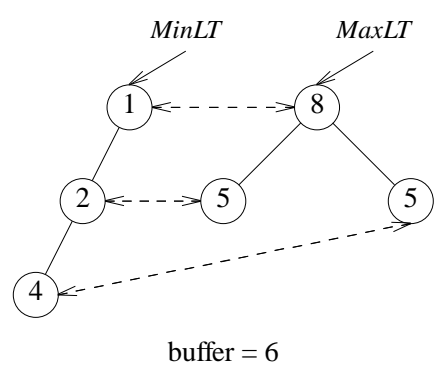

(a) Total correspondence

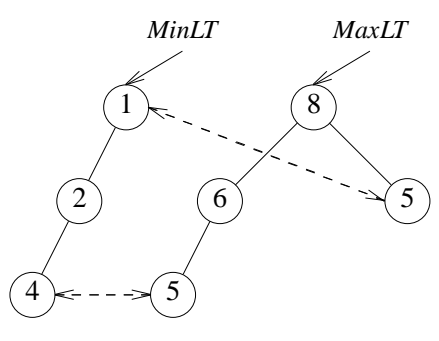

(b) Leaf correspondence

Broken arrows denote correspondence pointers Parent fields not shown

Fig. 3. Twin leftist trees

The storage requirements for the twin leftist tree adaptation are $4 n$ pointer fields, $n$ sh fields, and $n$ data fields (including that in the buffer).

\subsection{Algorithms For Total Correspondence}

To insert an element $e$ when the buffer is empty, we simply put $e$ into the buffer. When the buffer is not empty, the smaller of $e$ and the element in the buffer is inserted into MinLT and the larger into Max LT. Correspondence pointers between these two elements are also set up. Hence an insertion takes either $O(1)$ time (when the buffer is empty) and $O(\log n)$ time.

To merge two MDEPQs $Q 1$ and $Q 2$ to get the MDEPQ $Q 3$, the two min leftist trees $Q 1 . M i n L T$ and $Q 2 . M i n L T$ are merged to get $Q 3 . M i n L T$; the two max leftist 
trees Q1.MaxLT and Q2.MaxLT are merged to get Q3.MaxLT; Q3.buffer is set empty; the elements (if any) in Q1.buffer and Q2.buffer are inserted into Q3. Since two min $(\max )$ leftist trees can be merged in $O(\log n k)$ time and since an insertion into a total correspondence MDEPQ takes $O(\log n)$ time, the two MDEPQs are merged in $O(\log n k)$ time.

The DeleteMax operation may be implemented as in Figure 4. This requires that we be able to delete an arbitrary node $x$ from a min leftist tree. In Section 2 , we saw how this could be done in logarithmic time. So, the complexity of the DeleteMax algorithm of Figure 4 is $O(\log n)$. DeleteMin is similar.

Step 1: If the buffer is empty, do a max leftist tree DeleteMax on Q.MaxLT. Delete the corresponding node from Q.MinLT and put this element in the buffer. Return.

Step 2: [Buffer is not empty] If the element in the buffer has priority $\geq$ that of the one in the root of Q.MaxLT empty the buffer and return.

Step 3: Do a max leftist tree DeleteMax on Q.MaxLT. If the element in the buffer has priority $\geq$ that of the corresponding node, insert the buffer element into Q.MaxLT and set a correspondence between these two. If not, delete the corresponding node from Q.MinLT and insert it into Q.MaxLT; insert the buffer element into Q.MinLT; set a correspondence between the two.

Fig. 4. DeleteMax from a total correspondence twin leftist tree

\subsection{Algorithms For Leaf Correspondence}

With leaf correspondence twin leftist trees, we can avoid some of the work done when total correspondence leftist trees are used. For example, when inserting an element into an MDEPQ with a non empty buffer, it may be possible to get away with a single insert into the min leftist tree rather than an insert into both the min and max leftist trees (see Figure 5). The asymptotic complexity remains $O(\log n)$.

Step 1: If buffer is empty, put the new element $e$ into it and return.

Step 2: Let $b$ be the element in the buffer. Insert the smaller of $b$ and $e$ (call this element small) into Q.MinLT

Step 3: If small is not a leaf of Q.MinLT, put $\{b, e\}-$ small into the buffer and return.

Step 4: [small is a leaf] Insert $\{b, e\}-$ small into Q.MaxLT and set correspondence pointers between $b$ and $e$. Set the buffer to empty.

Fig. 5. Insertion into a leaf correspondence twin leftist tree

Two leaf correspondence twin leftist trees may be merged in $O(\log n k)$ time 
using the same procedure as used to merge two total correspondence leftist trees. To see that this works, we note that when two leftist trees are merged using the standard merge algorithm [8], no nonleaf node becomes a leaf. As a result, no new correspondences need to be established.

The DeleteMax algorithm is given in Figure 6. Like the DelteMax algorithm for total correspondence twin leftist trees, this makes a non-standard deletion from a leftist tree. This time, however, the non-standard deletions are restricted to leaf node deletions which can be done by a simple path traversal towards the root stopping when we reach a node whose $s h$ value doesn't change. The complexity of the algorithm of Figure 6 is $O(\log n)$. The DeleteMin algorithm is similar.

Step 1: [Empty buffer] If the buffer is empty, do a max leftist tree DeleteMax on Q.MaxLT.

If the correspondence pointer of the deleted node is null return.

If the corresponding node, $x$, is not a leaf of $Q \cdot \operatorname{Min} L T$, set its correspondence pointer to $N U L L$ and return.

Let $p$ be the parent (if any) of $x$ in MinLT.

Delete the leaf $x$ from $\operatorname{Min} L T$.

If $p$ has now become a leaf of MinLT and $p$ 's correspondence pointer is null, then insert $x$ into MaxLT and establish correspondence pointers between $p$ and $x$. Otherwise, put the element in $x$ into the buffer.

Return.

Step 2: [Buffer is not empty] If the element in the buffer has priority $\geq$ that of the one in the root of Q.MaxLT empty the buffer and return.

Step 3: Do a max leftist tree DeleteMax on Q.MaxLT.

If the correspondence pointer of the deleted node is null return.

If the corresponding node, $x$, is not a leaf of Q.MinLT, set its correspondence pointer to $N U L L$ and return.

If the buffer element, $b$, has priority $\geq$ that of $x$, insert it into $M a x L T$, establish correspondences pointers between $x$ and $b$, return.

Let $p$ be the parent (if any) of $x$ in MinLT.

Delete the leaf $x$ from $\operatorname{MinLT}$ and insert it into $\operatorname{Max} L T$.

If $p$ has now become a leaf of MinLT and $p$ 's correspondence pointer is null, then establish correspondence pointers between $p$ and $x$ and return.

If $x$ is a leaf, insert the buffer element, $b$, into MinLT and establish correspondence pointers between $x$ and $b$ and return.

Set $x$ 's correspondence pointer to null.

Fig. 6. DeleteMax from a leaf correspondence twin leftist tree

\section{Experimental Results}

We compared the performance of our second adaptation, the twin leftist tree (TLT), of leftist trees to that of other structures proposed for the representation of double-ended priority queues. Specifically, for the experimental comparison, we 
considered the array based structures min-max heaps (MMH) [2] and deaps (Deap) [4] as well as the pointer based structure min-max pair heap (MMP) [10]. Additionally, the pointer based dictionary structures unbalanced binary search tree (BST), AVL tree (AVL), and treap (TRP) [1] were adapted to directly support the deletion of the minimum and maximum elements. All of the structures were coded in $\mathrm{C}$ and run on a SUN SPARC-5. The code was compiled using the UNIX cc compiler in optimization mode. The AVL code was based on that of Ref. 11. The pointer codes saved deleted nodes on an internal chain so that calls to malloc were made only when an insert caused the number of elements to exceed the largest number of elements previously in the structure.

Since the TLT is the only structure in our test set that supports logarithmic merging of two double-ended priority queues, we know apriori that any experiment involving a large number of merge operations will favor the TLT structure. Instead, we focussed our experimental study on determining how well the TLT performs relative to the other structures when there are no merge operations. So, in the experiments, we began with a structure with $n$ elements for $n=100,1000$, and 10000 and performed a sequence of $m$ DEPQ operations (insert, delete max, and delete $\min )$. The choices for $m$ were 50,000, 100,000, and 200,000. For each $m$ a random sequence of insert, delete max, and delete min operations was also generated. The inserts were generated with probablity 0.5 and each type of delete operation had probability 0.25 . This tended to keep the structure size close to the initial value of $n$. The keys of the elements being inserted were generated in four different ways: (a) random $1 \cdots$ random keys in the range 1 through $10^{6}$; (b) random $2 \cdots$ random keys in the range 1 through 1000; (c) increasing keys; and (d) decreasing keys. Data set random 2 is expected to have many duplicate keys.

In our first experiment, we obtained the number of key comparisons performed by each of the methods. These are reported in Table 1 . The reported numbers for the data sets random 1 and random 2 are the average of ten runs. The standard deviation in the measured number of comparisons for these ten data sets is given in Table 2. For the increasing and decreasing data sets, the BST structure was excluded from the experiments as on these data BST's exhibit their worst performance and the run times are too large. As can be seen from the table, the TLT structure consistently performs fewer comparisons than any of the others. So, even when no merges are to be performed, the TLT is expected to outperform the other structures provided the cost of a key comparison is "sufficiently high".

While the key comparison results are independent of machine architecture, run time results are not. These are quite sensitive to the caching strategies, compiler optimization capabilities, relative cost of array and pointer accesses, etc. Our run time experiments were done once using integer keys and again using floating point keys. The data for the floating point case was the same as for the integer case except that the type of the key field was changed from int to float. The measured run times using integer keys are shown in Table 4 . As was the case for the number of comparisons, the times for the data sets random 1 and random 2 are the average of ten runs. The standard deviation in the measured times for these ten data sets 


\begin{tabular}{|c|c|c|c|c|c|c|c|c|c|}
\hline inputs & $m$ & $n$ & $\overline{\mathrm{BST}}$ & $\overline{\overline{\mathrm{MMH}}}$ & $\overline{\overline{\text { Deap }}}$ & $\overline{\overline{M M P}}$ & $\overline{\overline{\text { TLT }}}$ & 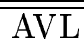 & $\overline{\overline{\text { TRP }}}$ \\
\hline & & 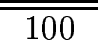 & 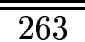 & 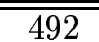 & $\begin{array}{c}307 \\
\end{array}$ & $\begin{array}{c}397 \\
\end{array}$ & $\overline{c 138}$ & 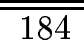 & 202 \\
\hline & 50,000 & 1000 & 311 & 837 & 465 & 556 & 171 & 272 & 282 \\
\hline & & 10000 & 384 & 1148 & 547 & 634 & 372 & 349 & 405 \\
\hline \multirow{9}{*}{ random1 } & \multirow{3}{*}{100,000} & 100 & 492 & 961 & 601 & 783 & 267 & 363 & 403 \\
\hline & & 1000 & 649 & 1662 & 932 & 1114 & 314 & 545 & 546 \\
\hline & & 10000 & 709 & 2329 & 1165 & 1342 & 603 & 693 & 746 \\
\hline & \multirow{3}{*}{200,000} & 100 & 1125 & 2119 & 1298 & 1662 & 538 & 776 & 853 \\
\hline & & 1000 & 1412 & 3405 & 1914 & 2278 & 593 & 1103 & 1068 \\
\hline & & 10000 & 1408 & 4670 & 2433 & 2792 & 981 & 1379 & 1402 \\
\hline & \multirow{3}{*}{50,000} & $\bar{~} 100$ & $\overline{233}$ & $\overline{4439}$ & $\overline{2811}$ & $\overline{372}$ & $\overline{130}$ & $\overline{172}$ & $\overline{194}$ \\
\hline & & 1000 & 368 & 805 & 454 & 543 & 167 & 268 & 281 \\
\hline & & 10000 & 607 & 1117 & 553 & 639 & 349 & 351 & 444 \\
\hline \multirow{9}{*}{ random 2} & \multirow{3}{*}{100,000} & 100 & 539 & 988 & 615 & 796 & 265 & 368 & 411 \\
\hline & & 1000 & 855 & 1660 & 939 & 1119 & 309 & 536 & 574 \\
\hline & & 10000 & 1326 & 2235 & 1182 & 1354 & 571 & 692 & 871 \\
\hline & \multirow{3}{*}{200,000} & 100 & 1247 & 2082 & 1281 & 1643 & 533 & 765 & 837 \\
\hline & & 1000 & 2489 & 3372 & 1910 & 2271 & 584 & 1064 & 1148 \\
\hline & & 10000 & 3318 & 4502 & 2475 & 2822 & 922 & 1362 & 1785 \\
\hline & \multirow{3}{*}{50,000} & 100 & - & $\overline{446}$ & 322 & $\overline{409}$ & $\overline{97}$ & $\overline{178}$ & $\overline{179}$ \\
\hline & & 1000 & - & 821 & 547 & 634 & 100 & 282 & 251 \\
\hline & & 10000 & - & 1196 & 753 & 839 & 100 & 363 & 306 \\
\hline \multirow{9}{*}{ decreasing } & \multirow{3}{*}{100,000} & 100 & - & 976 & 688 & 862 & 196 & 375 & 371 \\
\hline & & 1000 & - & 1661 & 1107 & 1280 & 200 & 563 & 502 \\
\hline & & 10000 & - & 2395 & 1509 & 1683 & 200 & 725 & 656 \\
\hline & \multirow{3}{*}{200,000} & 100 & - & 1980 & 1392 & 1741 & 392 & 756 & 747 \\
\hline & & 1000 & - & 3341 & 2229 & 2575 & 400 & 1123 & 998 \\
\hline & & 10000 & - & 4789 & 3031 & 3378 & 400 & 1451 & 1255 \\
\hline & \multirow{3}{*}{50,000} & 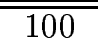 & 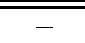 & $\overline{5525}$ & $\overline{373}$ & $\overline{4462}$ & $\overline{c 99}$ & $\overline{195}$ & $\overline{190}$ \\
\hline & & 1000 & - & 860 & 567 & 656 & 100 & 283 & 252 \\
\hline & & 10000 & - & 1199 & 766 & 854 & 100 & 363 & 302 \\
\hline \multirow{6}{*}{ increasing } & \multirow{3}{*}{100,000} & 100 & $\overline{-}$ & 1046 & 743 & 921 & 197 & 387 & 375 \\
\hline & & 1000 & - & 1736 & 1144 & 1321 & 200 & 563 & 495 \\
\hline & & 10000 & - & 2399 & 1535 & 1712 & 200 & 726 & 631 \\
\hline & \multirow{3}{*}{200,000} & 100 & - & 1936 & 1397 & 1753 & 391 & 736 & 735 \\
\hline & & 1000 & - & 3429 & 2265 & 2620 & 399 & 1126 & 1003 \\
\hline & & 10000 & - & 4800 & 3081 & 3436 & 399 & 1451 & 1248 \\
\hline
\end{tabular}

$m=$ the number of operations performed

$n=$ the number of elements in initial data structures

Table 1. The number of key comparisons (in thousands) 


\begin{tabular}{|c|c|c||c|c|c|c|c|c|c|}
\hline \hline inputs & $m$ & $n$ & BST & MMH & Deap & MMP & TLT & AVL & TRP \\
\hline \hline & \multirow{3}{*}{50,000} & 100 & 37171 & 49653 & 23503 & 23623 & 2947 & 13065 & 10892 \\
& & 10000 & 13485 & 16765 & 8177 & 8196 & 1786 & 2106 & 9250 \\
& & 100 & 1036 & 418 & 481 & 578 & 139 & 491 & 2424 \\
random1 & \multirow{2}{*}{100,000} & 1000 & 12643 & 623 & 451 & 630 & 328 & 2432 & 5451 \\
& & 10000 & 4421 & 69 & 197 & 308 & 375 & 1799 & 9020 \\
\hline & \multirow{2}{*}{200,000} & 100 & 7770 & 1174 & 485 & 659 & 144 & 93 & 614 \\
& & 1000 & 14384 & 886 & 73 & 343 & 497 & 296 & 5254 \\
& & 10000 & 2437 & 112 & 120 & 79 & 296 & 4424 & 2283 \\
\hline \hline & \multirow{3}{*}{50,000} & 100 & 723 & 1188 & 602 & 600 & 285 & 385 & 2223 \\
& & 1000 & 11047 & 321 & 267 & 260 & 205 & 1369 & 4903 \\
& & 10000 & 9505 & 1013 & 183 & 183 & 535 & 1159 & 7506 \\
\hline \multirow{3}{*}{ random2 } & 100,000 & 1000 & 11281 & 91 & 150 & 259 & 313 & 406 & 1792 \\
& & 10000 & 3040 & 203 & 172 & 119 & 331 & 473 & 15151 \\
& \multirow{3}{*}{200,000} & 100 & 5399 & 86 & 222 & 217 & 384 & 193 & 2503 \\
& & 1000 & 21103 & 1125 & 263 & 196 & 194 & 3458 & 17357 \\
& & 10000 & 21495 & 68 & 172 & 279 & 287 & 1307 & 11545 \\
\hline \hline
\end{tabular}

$m=$ the number of operations performed

$n=$ the number of elements in initial data structures

Table 2. Standard deviation of the number of key comparisons 
is given in Table 4. The corresponding times using floating point keys are given in Table 5 and 6 . Once again, for the increasing and decreasing data sets, the BST structure was excluded from the experiments as on these data BST's exhibit their worst performance and the run times are too large.

Table 4 shows that the BST has best performance on the random1 data set. For the random2 data set, BST is best when $n=100$. For the remaining values of $n$, BST and TLT were tied on one data set, TLT was best on four, and Deap was best on two. On the increasing and decreasing data sets, TLT was consistently best. Since the BST is very slow on ordered data (i.e., increasing or decreasing), it is not recommended for general applications. So, it is worthwhile to see which structure did second best the times when BST was best. The TLT held second palce on 11 of these data sets and the Deap did this on the remaining three.

The change to floating point keys increased the cost of a key comparison. However, this increase was not enough for TLT to outperform BST on the random1 data set. BST remained best on seven of the eight data sets and TLT was best on one. On the random2 data set, there was no clear winner. The BST, TLT, AVL, TRP data structures were competitive. The TLT remained consistently superior of the decreasing data set and was generally best on the increasing data. On this latter data set, the randomized structure TRP tied the TLT three times and was faster twice.

\section{Conclusion}

We have proposed two adaptations of the leftist tree structure to support mergeable double-ended priority queues. Both permit one to perform each of the operations: insert, delete max, delete min, and merge in logarithmic time. For applications in which good amortized complexity suffices, the Fibonacci heap data structure described in Refs. 8,12 may be adapted to obtain a twin Fibonacci heap. Using this data structure, inserts and merges can be done in $\Theta(1)$ time while delete min and delete max have amortized complexity $O(\log n)$. The second of our adaptations, the twin leftist tree, is more space efficient than the first (dual leftist tree). Experimental results obtained by us indicate that the twin leftist tree is actually more efficient (i.e., faster) than data structures (e.g., Deap and $\mathrm{MMH}$ ) previously proposed for non-mergeable double-ended priority queues.

\section{References}

1. C. R. Aragon and R. G. Seidel, Randomized Search Trees, Proc. 30th Ann. IEEE Symposium on Foundations of Computer Science, pp. 540-545, October 1989.

2. M. Atkinson, J. Sack, N. Santoro, and T. Strothotte, Min-max heaps and generalized priority queues, Communications of the ACM, 29, 996-1000, 1986.

3. G. Brodal, Fast meldable priority queues, Workshop on Algorithms and Data Structures, 1995.

4. S. Carlsson, The deap - A double ended heap to implement double ended priority queues, Information Processing Letters, 26, 33-36, 1987.

5. S. Chang and M. Du, Diamond deque: A simple data structure for priority deques, 


\begin{tabular}{|c|c|c|c|c|c|c|c|c|c|}
\hline inputs & $m$ & $n$ & BST & MMH & Deap & MMP & TLT & AVL & TRP \\
\hline & & 100 & 0.11 & 0.20 & 0.14 & 0.13 & 0.12 & 0.18 & 0.16 \\
\hline & 50,000 & 1000 & 0.12 & 0.29 & 0.20 & 0.20 & 0.16 & 0.22 & 0.19 \\
\hline & & 10000 & 0.16 & 0.39 & 0.24 & 0.29 & 0.35 & 0.28 & 0.28 \\
\hline \multirow{9}{*}{ random1 } & \multirow{3}{*}{100,000} & 100 & 0.21 & 0.38 & 0.28 & 0.27 & 0.24 & 0.36 & 0.31 \\
\hline & & 1000 & 0.26 & 0.58 & 0.39 & 0.38 & 0.28 & 0.43 & 0.37 \\
\hline & & 10000 & 0.31 & 0.79 & 0.49 & 0.56 & 0.58 & 0.54 & 0.50 \\
\hline & \multirow{3}{*}{200,000} & 100 & $\overline{0.45}$ & 0.81 & 0.58 & 0.56 & 0.49 & 0.73 & 0.64 \\
\hline & & 1000 & 0.55 & 1.19 & 0.79 & 0.77 & 0.55 & 0.87 & 0.73 \\
\hline & & 10000 & 0.60 & 1.57 & 0.99 & 1.08 & 0.96 & 1.05 & 0.93 \\
\hline & \multirow{3}{*}{50,000} & 100 & $\overline{0.10}$ & $\overline{0.18}$ & $\overline{0.13}$ & $\overline{0.13}$ & $\overline{0.12}$ & $\bar{~} 0.17$ & $\overline{0.15}$ \\
\hline & & 1000 & 0.15 & 0.28 & 0.19 & 0.19 & 0.15 & 0.22 & 0.18 \\
\hline & & 10000 & 0.27 & 0.38 & 0.25 & 0.29 & 0.33 & 0.29 & 0.31 \\
\hline \multirow{9}{*}{ random2 } & \multirow{3}{*}{100,000} & 100 & 0.22 & 0.38 & 0.28 & 0.27 & 0.24 & 0.36 & 0.32 \\
\hline & & 1000 & 0.33 & 0.59 & 0.40 & 0.38 & 0.28 & 0.44 & 0.38 \\
\hline & & 10000 & 0.57 & 0.75 & 0.49 & 0.57 & 0.55 & 0.55 & 0.58 \\
\hline & \multirow{3}{*}{200,000} & 100 & 0.48 & 0.80 & 0.58 & 0.55 & 0.49 & 0.72 & 0.63 \\
\hline & & 1000 & 0.91 & 1.17 & 0.78 & 0.77 & 0.55 & 0.86 & 0.75 \\
\hline & & 10000 & 1.38 & 1.51 & 1.01 & 1.11 & 0.90 & 1.06 & 1.13 \\
\hline & \multirow{3}{*}{50,000} & 100 & - & 0.18 & 0.15 & 0.13 & 0.08 & 0.17 & 0.15 \\
\hline & & 1000 & - & 0.30 & 0.23 & 0.22 & 0.10 & 0.20 & 0.18 \\
\hline & & 10000 & - & 0.40 & 0.28 & 0.28 & 0.10 & 0.23 & 0.20 \\
\hline \multirow{9}{*}{ decreasing } & \multirow{3}{*}{100,000} & 100 & - & 0.38 & 0.30 & 0.27 & 0.20 & 0.32 & 0.32 \\
\hline & & 1000 & - & 0.60 & 0.45 & 0.48 & 0.20 & 0.45 & 0.40 \\
\hline & & 10000 & - & 0.82 & 0.57 & 0.60 & 0.20 & 0.48 & 0.40 \\
\hline & \multirow{3}{*}{200,000} & 100 & - & 0.80 & 0.62 & 0.57 & 0.37 & 0.65 & 0.62 \\
\hline & & 1000 & - & 1.22 & 0.88 & 0.93 & 0.40 & 0.83 & 0.73 \\
\hline & & 10000 & - & 1.65 & 1.17 & 1.23 & 0.40 & 0.93 & 0.78 \\
\hline & \multirow{3}{*}{50,000} & 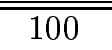 & - & 0.20 & 0.17 & 0.15 & 0.10 & 0.17 & 0.17 \\
\hline & & 1000 & - & 0.30 & 0.23 & 0.23 & 0.10 & 0.22 & 0.18 \\
\hline & & 10000 & - & 0.42 & 0.30 & 0.28 & 0.12 & 0.25 & 0.22 \\
\hline \multirow{6}{*}{ increasing } & \multirow{3}{*}{100,000} & 100 & - & 0.42 & $\overline{0.32}$ & 0.28 & $\overline{0.22}$ & 0.33 & 0.32 \\
\hline & & 1000 & - & 0.63 & 0.45 & 0.45 & 0.23 & 0.43 & 0.35 \\
\hline & & 10000 & - & 0.83 & 0.58 & 0.60 & 0.22 & 0.50 & 0.40 \\
\hline & \multirow{3}{*}{200,000} & 100 & - & 0.80 & 0.60 & 0.53 & 0.43 & 0.67 & 0.62 \\
\hline & & 1000 & - & 1.25 & 0.92 & 0.92 & 0.48 & 0.85 & 0.72 \\
\hline & & 10000 & - & 1.68 & 1.18 & 1.22 & 0.47 & 0.95 & 0.78 \\
\hline
\end{tabular}

Time Unit : sec

$m=$ the number of operations performed

$n=$ the number of elements in initial data structures

Table 3. Run time using integer keys 


\begin{tabular}{|c|c|c|c|c|c|c|c|c|c|}
\hline inputs & $m$ & $n$ & $\overline{B S T}$ & MMH & Deap & MMP & TLT & AVL & $\overline{\text { TRP }}$ \\
\hline & & 100 & 0.015 & 0.015 & 0.013 & 0.009 & 0.008 & $\overline{0.013}$ & 0.008 \\
\hline & 50,000 & 1000 & 0.008 & 0.011 & 0.007 & 0.007 & 0.011 & 0.007 & 0.011 \\
\hline & & 10000 & 0.009 & 0.008 & 0.008 & 0.008 & 0.005 & 0.011 & 0.012 \\
\hline \multirow{9}{*}{ random1 } & \multirow{3}{*}{100,000} & 100 & 0.008 & 0.007 & 0.008 & 0.009 & 0.008 & 0.008 & 0.007 \\
\hline & & 1000 & 0.009 & 0.005 & 0.008 & 0.008 & 0.009 & 0.007 & 0.010 \\
\hline & & 10000 & 0.008 & 0.010 & 0.011 & 0.008 & 0.007 & 0.011 & 0.008 \\
\hline & \multirow{3}{*}{200,000} & 100 & 0.000 & 0.008 & 0.009 & 0.011 & 0.011 & 0.014 & 0.011 \\
\hline & & 1000 & 0.007 & 0.008 & 0.010 & 0.008 & 0.009 & 0.008 & 0.007 \\
\hline & & 10000 & 0.008 & 0.013 & 0.011 & 0.005 & 0.011 & 0.009 & 0.009 \\
\hline & \multirow{3}{*}{50,000} & 100 & 0.005 & 0.008 & 0.009 & 0.007 & 0.000 & 0.008 & 0.008 \\
\hline & & 1000 & 0.005 & 0.005 & 0.008 & 0.010 & 0.005 & 0.011 & 0.009 \\
\hline & & 10000 & 0.007 & 0.000 & 0.007 & 0.008 & 0.011 & 0.008 & 0.008 \\
\hline \multirow{6}{*}{ random2 } & \multirow{3}{*}{100,000} & 100 & 0.009 & 0.007 & 0.007 & 0.007 & 0.008 & 0.008 & 0.017 \\
\hline & & 1000 & 0.012 & 0.013 & 0.007 & 0.005 & 0.009 & 0.015 & 0.011 \\
\hline & & 10000 & 0.008 & 0.007 & 0.008 & 0.009 & 0.007 & 0.007 & 0.011 \\
\hline & \multirow{3}{*}{200,000} & 100 & 0.008 & 0.009 & 0.013 & 0.000 & 0.007 & 0.011 & 0.005 \\
\hline & & 1000 & 0.015 & 0.011 & 0.005 & 0.008 & 0.010 & 0.010 & 0.012 \\
\hline & & 10000 & 0.008 & 0.005 & 0.016 & 0.015 & 0.000 & 0.008 & 0.011 \\
\hline
\end{tabular}

Time Unit : sec

$m=$ the number of operations performed

$n=$ the number of elements in initial data structures

Table 4. Standard deviation of run time using integer keys 


\begin{tabular}{|c|c|c|c|c|c|c|c|c|c|}
\hline inputs & $m$ & $n$ & BST & MMH & Deap & MMP & TLT & AVL & TRP \\
\hline & & 100 & 0.15 & 0.28 & 0.21 & 0.21 & 0.16 & 0.19 & 0.15 \\
\hline & 50,000 & 1000 & 0.18 & 0.44 & 0.30 & 0.33 & 0.21 & 0.25 & 0.20 \\
\hline & & 10000 & 0.24 & 0.60 & 0.37 & 0.46 & 0.48 & 0.33 & 0.33 \\
\hline \multirow{9}{*}{ random1 } & \multirow{3}{*}{100,000} & 100 & 0.28 & 0.56 & 0.41 & 0.41 & 0.33 & 0.39 & 0.31 \\
\hline & & 1000 & 0.37 & 0.88 & 0.59 & 0.65 & 0.39 & 0.49 & 0.39 \\
\hline & & 10000 & 0.44 & 1.20 & 0.75 & 0.90 & 0.79 & 0.64 & 0.59 \\
\hline & \multirow{3}{*}{200,000} & 100 & 0.63 & 1.20 & 0.86 & 0.87 & 0.67 & 0.81 & 0.63 \\
\hline & & 1000 & 0.80 & 1.81 & 1.21 & 1.30 & 0.75 & 0.99 & 0.76 \\
\hline & & 10000 & 0.85 & 2.39 & 1.52 & 1.71 & 1.31 & 1.23 & 1.06 \\
\hline & \multirow{3}{*}{50,000} & 100 & $\overline{0.14}$ & 0.26 & 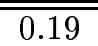 & "0.20 & "0.16 & "0.18 & 0.15 \\
\hline & & 1000 & 0.20 & 0.43 & 0.29 & 0.32 & 0.21 & 0.25 & 0.20 \\
\hline & & 10000 & 0.40 & 0.59 & 0.37 & 0.47 & 0.45 & 0.34 & 0.36 \\
\hline \multirow{9}{*}{ random2 } & \multirow{3}{*}{100,000} & 100 & 0.31 & 0.56 & 0.41 & 0.42 & 0.33 & 0.40 & 0.31 \\
\hline & & 1000 & 0.47 & 0.89 & 0.60 & 0.66 & 0.39 & 0.49 & 0.40 \\
\hline & & 10000 & 0.84 & 1.17 & 0.77 & 0.92 & 0.76 & 0.65 & 0.68 \\
\hline & \multirow{3}{*}{200,000} & 100 & 0.68 & 1.18 & 0.85 & 0.87 & 0.66 & 0.80 & 0.63 \\
\hline & & 1000 & 1.34 & 1.79 & 1.22 & 1.29 & 0.75 & 0.99 & 0.80 \\
\hline & & 10000 & 2.07 & 2.32 & 1.55 & 1.77 & 1.24 & 1.28 & 1.32 \\
\hline & \multirow{3}{*}{50,000} & 100 & - & 0.27 & 0.20 & 0.23 & 0.12 & 0.20 & 0.15 \\
\hline & & 1000 & - & 0.48 & 0.33 & 0.38 & 0.13 & 0.25 & 0.17 \\
\hline & & 10000 & - & 0.63 & 0.45 & 0.47 & 0.13 & 0.28 & 0.20 \\
\hline \multirow{9}{*}{ decreasing } & \multirow{3}{*}{100,000} & 100 & - & 0.63 & 0.47 & 0.50 & 0.25 & 0.40 & 0.33 \\
\hline & & 1000 & - & 1.00 & 0.70 & 0.85 & 0.25 & 0.53 & 0.40 \\
\hline & & 10000 & - & 1.28 & 0.88 & 0.97 & 0.27 & 0.57 & 0.43 \\
\hline & \multirow{3}{*}{200,000} & 100 & $\overline{-}$ & 1.18 & 0.90 & 0.92 & 0.50 & 0.78 & 0.60 \\
\hline & & 1000 & - & 1.88 & 1.37 & 1.62 & 0.52 & 1.00 & 0.75 \\
\hline & & 10000 & - & 2.58 & 1.80 & 2.02 & 0.53 & 1.13 & 0.87 \\
\hline & \multirow{3}{*}{50,000} & 100 & - & 0.32 & 0.23 & 0.25 & 0.15 & 0.22 & 0.15 \\
\hline & & 1000 & - & 0.48 & 0.35 & 0.40 & 0.18 & 0.25 & 0.18 \\
\hline & & 10000 & - & 0.63 & 0.45 & 0.47 & 0.20 & 0.30 & 0.20 \\
\hline \multirow{6}{*}{ increasing } & \multirow{3}{*}{100,000} & 100 & $\overline{-}$ & $\overline{0.60}$ & 0.47 & 0.48 & 0.33 & 0.40 & 0.30 \\
\hline & & 1000 & - & 0.97 & 0.70 & 0.82 & 0.35 & 0.52 & 0.38 \\
\hline & & 10000 & - & 1.28 & 0.92 & 0.97 & 0.35 & 0.57 & 0.43 \\
\hline & \multirow{3}{*}{200,000} & 100 & - & 1.18 & 0.90 & 0.92 & 0.65 & 0.78 & 0.60 \\
\hline & & 1000 & - & 1.93 & 1.57 & 1.58 & 0.70 & 1.02 & 0.73 \\
\hline & & 10000 & - & 2.58 & 1.83 & 1.98 & 0.68 & 1.13 & 0.83 \\
\hline
\end{tabular}

Time Unit : sec

$m=$ the number of operations performed

$n=$ the number of elements in initial data structures

Table 5. Run time using real (double) keys 


\begin{tabular}{|c|c|c|c|c|c|c|c|c|c|}
\hline inputs & $m$ & $n$ & $\overline{B S T}$ & MMH & Deap & MMP & TLT & AVL & TRP \\
\hline & & 100 & $\overline{0.014}$ & $\overline{0.021}$ & 0.011 & 0.015 & 0.009 & $\overline{0.008}$ & $\overline{0.008}$ \\
\hline & 50,000 & 1000 & 0.011 & 0.011 & 0.012 & 0.011 & 0.008 & 0.007 & 0.011 \\
\hline & & 10000 & 0.008 & 0.005 & 0.007 & 0.010 & 0.007 & 0.000 & 0.012 \\
\hline \multirow{9}{*}{ random1 } & \multirow{3}{*}{100,000} & 100 & 0.007 & 0.011 & 0.008 & 0.008 & 0.005 & 0.011 & 0.008 \\
\hline & & 1000 & 0.010 & 0.000 & 0.008 & 0.007 & 0.011 & 0.008 & 0.011 \\
\hline & & 10000 & 0.010 & 0.000 & 0.010 & 0.005 & 0.008 & 0.011 & 0.023 \\
\hline & \multirow{3}{*}{200,000} & 100 & 0.009 & 0.010 & 0.008 & 0.008 & 0.015 & 0.011 & 0.009 \\
\hline & & 1000 & 0.018 & 0.011 & 0.009 & 0.010 & 0.007 & 0.008 & 0.008 \\
\hline & & 10000 & 0.009 & 0.008 & 0.008 & 0.007 & 0.015 & 0.007 & 0.011 \\
\hline & \multirow{3}{*}{50,000} & 100 & 0.008 & 0.008 & 0.008 & 0.010 & 0.008 & 0.009 & 0.005 \\
\hline & & 1000 & 0.010 & 0.008 & 0.008 & 0.011 & 0.008 & 0.007 & 0.007 \\
\hline & & 10000 & 0.010 & 0.008 & 0.008 & 0.008 & 0.008 & 0.008 & 0.008 \\
\hline \multirow{6}{*}{ random2 } & \multirow{3}{*}{100,000} & 100 & 0.008 & 0.010 & 0.007 & 0.008 & 0.010 & 0.007 & 0.009 \\
\hline & & 1000 & 0.019 & 0.008 & 0.007 & 0.012 & 0.008 & 0.008 & 0.010 \\
\hline & & 10000 & 0.008 & 0.011 & 0.008 & 0.011 & 0.008 & 0.011 & 0.018 \\
\hline & \multirow{3}{*}{200,000} & 100 & 0.005 & 0.012 & 0.007 & 0.007 & 0.005 & 0.005 & 0.009 \\
\hline & & 1000 & 0.012 & 0.008 & 0.009 & 0.008 & 0.005 & 0.008 & 0.016 \\
\hline & & 10000 & 0.017 & 0.008 & 0.005 & 0.011 & 0.011 & 0.008 & 0.013 \\
\hline
\end{tabular}

Time Unit : sec

$m=$ the number of operations performed

$n=$ the number of elements in initial data structures

Table 6. Standard deviation of run time using real keys 
Information Processing Letters, 46, 231-237, 1993.

6. C. Crane, Linear lists and priority queues as balanced binary trees, Technical Report CS-72-259, Computer Science Department, Stanford University, CA, 1972.

7. A. Hasham and J. Sack, Bounds for min-max heaps, BIT, 27, 315-323, 1987.

8. E. Horowitz, S. Sahni, D. Mehta, Fundamentals of Data Structures in $\mathrm{C}++$, Computer Science Press, NY, 1995.

9. J. van Leeuwen and D. Wood, Interval heaps, The Computer Journal, 36, 3, 209-216, 1993.

10. S. Olariu, C. Overstreet, and Z. Wen, A mergeable double-ended priority queue, The Computer Journal, 34, 5, 423-427, 1991.

11. T. Papadakis, Skip Lists and Probabilistic Analysis of Algorithms, PhD Dissertation, Univ. of Waterloo, 1993.

12. R. Tarjan, Data structures and network algorithms, SIAM, Philadelphia, PA, 1983.

13. J. Williams, Algorithm 232, Communications of the ACM, 7, 347-348, 1964. 